\title{
Analytical model of static coronal loops
}

\author{
J. Dudík ${ }^{1}$, E. Dzifčáková ${ }^{1,2}$, M. Karlický ${ }^{2}$, and A. Kulinováa ${ }^{1,2}$ \\ 1 Department of Astronomy, Physics of the Earth and Meteorology, Faculty of Mathematics, Physics and Informatics, Comenius \\ University, Mlynská Dolina F2, 84248 Bratislava, Slovak Republic \\ e-mail: dudik@fmph.uniba.sk \\ 2 Astronomical Institute of the Academy of Sciences of the Czech Republic, Fričova 298, 25165 Ondřejov, Czech Republic \\ Received 9 September 2008 / Accepted 23 March 2009
}

\section{ABSTRACT}

\begin{abstract}
By solving the energy-equilibrium equation in the stationary case, we derive analytical formulae in the form of scaling laws for non-uniformly heated and gravitationally stratified coronal loops. The heating is assumed to be localized in the chromosphere and to exponentially decrease with increasing distance along the loop strand. This exponential behavior of the heating and pressure profiles implies that we need to use the mean-value theorem, and in turn fit the mean-value parameters of the scaling laws to the results of the numerical simulations. The radiative-loss function is approximated by a power-law function of the temperature, and its effect on the resulting scaling laws for coronal loops is studied. We find that this effect is more important than the effect of varying loop geometry. We also find that the difference in lengths of the different loop strands in a loop with expanding cross-section does not produce differences in the EUV emission of these strands significant enough to explain the observed narrowness of the coronal loops.
\end{abstract}

Key words. hydrodynamics - Sun: corona - Sun: UV radiation - stars: coronae

\section{Introduction}

Since the first observations of the solar corona in the extreme ultraviolet (EUV) and soft X-rays, it has been undeniably clear that the corona is a highly structured environment. The basic structural blocks observed mainly in active regions are coronal loops - thin, arch-like, or elongated cylindrical structures delineating magnetic field lines. Coronal loops are anchored in the solar chromosphere at one or both ends. The presence of EUV and $\mathrm{X}$-ray emission from these coronal loops means that the loops are hot - their temperatures reach several million $\mathrm{K}$. The fact that the physical mechanism(s) heating the corona has still not been successfully identified constitutes the coronal heating problem (e.g., Klimchuk 2006).

One approach to constraining the coronal heating problem is the direct physical modeling of the temperature and density structure of the coronal loops. Analytical models are an invaluable tool for accomplishing this task, even if they employ a number of simplifying assumptions. The most frequent assumption is that of static energy equilibrium. Further assumptions include the uniform pressure throughout the loop (e.g., Rosner et al. 1978; Craig et al. 1978; Chiuderi et al. 1981; Kuin \& Martens 1982; Martens 2009) and uniform energy input (Vesecky et al. 1979), while others relax one or both of these assumptions (e.g., Wragg \& Priest 1979; Serio et al. 1981; Aschwanden \& Schrijver 2002). The stability of the static solutions have been extensively studied (e.g., Antiochos 1979; Hood \& Priest 1979, 1980; Chiuderi et al. 1981; Craig et al. 1982). Aschwanden \& Tsiklauri (2009) demonstrated the feasibility of analytical models even for non-static situations corresponding to impulsively heated loops with subsequent cooling.

The usual solution to the energy balance equation in the static case is written in the form of scaling laws due to the over-specification of the boundary conditions (Martens 2009). The three most seminal papers on scaling laws are those of Rosner et al. (1978), Serio et al. (1981), and
Aschwanden \& Schrijver (2002). The first two of these papers assume a simplified radiative-loss function in the form of the temperature power-law with fixed parameters, while the latest uses the radiative-loss function of Tucker \& Koren (1971). The effect of the radiative-loss function parameters on resulting scaling laws was studied by Kuin \& Martens (1982) and Martens (2009), although only for the loops with uniform pressure. None of these papers treat the effects of varying loop geometry. However, the effect of the varying loop cross-sectional area on the temperature and density profiles of the coronal loops has been extensively studied (Vesecky et al. 1979; Aschwanden \& Schrijver 2002; Martens 2009) and found to be small, even though the changes in temperature and density profiles act cumulatively to increase the differential emission measure near the loop apex (Vesecky et al. 1979).

We derive new analytical scaling laws for non-uniformly heated and gravitationally stratified coronal loops. These scaling laws are explicitly dependent on the parameters of the radiativeloss function, making them the most general scaling laws for coronal loops existing to date.

This paper is organized as follows. The assumptions and derivation of new scaling laws for coronal loops is given in Sect. 2. Discussion of different effects on the equilibrium solutions or resulting EUV emission is given in Sect. 3, which includes studies of the effect of the loop geometry (Sect. 3.2), the effect of the radiative-loss function parameters (Sect. 3.3), and the effect of the expanding loop cross-section on the EUV emission in a given TRACE filter (Sect. 3.5). Our conclusions are summarized in Sect. 4.

\section{Hydrostatic scaling laws}

\subsection{Hydrostatic equations and assumptions}

To construct an analytical model of a coronal loop, several assumptions about the nature of energy equilibrium, chemical 
composition, and gravitational stratification of the solar corona must be made. These assumptions are discussed below.

For stellar coronae, the gas pressure is significantly higher than the radiation pressure. The total kinetic pressure $p$ is then related to the electron density $n_{\mathrm{e}}$ and (electron) temperature $T$ by the equation of state

$p=q n_{\mathrm{e}} k_{\mathrm{B}} T$,

where $k_{\mathrm{B}} \approx 1.38 \times 10^{-23} \mathrm{JK}^{-1}$ is the Boltzmann constant and $q$ is the particle fraction number per one electron. The value of $q$ is given by the chemical composition and ionization equilibrium in the star's corona. In the simplest case of fully ionized hydrogen plasma, $q=2$. This value has been used by previous authors (Rosner et al. 1978; Serio et al. 1981; Aschwanden \& Schrijver 2002).

The hydrostatic balance equation

$\frac{\mathrm{d} p(h)}{\mathrm{d} h}=-\tilde{\mu} m_{\mathrm{H}} n_{\mathrm{e}}(h) g_{\odot}\left(1+\frac{h}{R_{\odot}}\right)^{-2}$

together with the equation of state in Eq. (1) gives the pressure stratification of the solar atmosphere. Here $R_{\odot}$ and $g_{\odot}$ represents the solar radius and surface gravity, respectively, $m_{\mathrm{H}}=1.67 \times$ $10^{-27} \mathrm{~kg}$ is the mass of a hydrogen atom, and $\tilde{\mu}$ is the hydrogen mass fraction per one electron. The last quantity is related to the mean molecular weight $\mu$ as $\mu=\tilde{\mu} / q$. For solar corona, $\tilde{\mu} \sim 1.3$. The pressure $p$ at height $h$ above the solar photosphere is given by the solution of Eq. (2)

$p(h)=p_{0}\left(h=h_{0}\right) \exp \left(-\int_{h_{0}}^{h} \frac{\mathrm{d} x}{\lambda_{\mathrm{p}}(T(x))\left(1+x / R_{\odot}\right)^{2}}\right)$.

Here $h_{0}$ denotes the height of the upper chromosphere transition-region boundary and $\lambda_{\mathrm{p}}$ is the pressure scale height

$\lambda_{\mathrm{p}}(h)=\lambda_{0} T(h)=\frac{k_{\mathrm{B}} T(h)}{\mu m_{\mathrm{H}} g_{\odot}}$.

If the near-isothermal approximation $(T(h) \approx$ constant $)$ holds, the previous equation reduces to

$p(h)=p_{0}\left(h_{0}\right) \exp \left(-\frac{h-h_{0}}{\lambda_{\mathrm{p}}(h)\left(1+h / R_{\odot}\right)\left(1+h_{0} / R_{\odot}\right)}\right)$,

where the term $\left(1+h_{0} / R_{\odot}\right)$ is usually very close to unity, since $h_{0} \ll R_{\odot}$.

For a non-isothermal atmosphere, the following analytical approximation is applicable (Aschwanden \& Schrijver 2002)

$p(h)=p_{0}\left(h_{0}\right) \exp \left(-\frac{h-h_{0}}{\lambda_{\mathrm{p}}(h)\left(1+h / R_{\odot}\right) q_{\lambda}}\right)$,

where the correction factor $q_{\lambda}$ is in general a function of the temperature and also other loop parameters, but is not a function of the height $h$ (Aschwanden \& Schrijver 2002, Eqs. (26) and (27)).

Since the magnetic field usually dominates the force balance in the solar corona, it can be safely assumed that the cross-field transportation effects are negligible and the frozen-in and forcefree approximations hold well in this environment. Hydrostatic balance along a given magnetic field line (loop strand; Martens 2009) can then be expressed in terms of the pressure and temperature dependence $p=p(s), T=T(s)$, where $s$ is the position along the field line, measured upwards from the photospheric footpoint $s=0$ to the highest position on the field line (apex) $s_{1}$.
The correspondence between $p(s)$ and $p(h)$ is given by the field line geometry, i.e., the mapping $s \leftrightarrow h$.

The energy balance along a magnetic field line is determined by the balance of energy sources and sinks. In the stationary case it is given by the equilibrium between radiative losses $E_{\mathrm{R}}$, heating sources $E_{\mathrm{H}}$, and the divergence of thermal conductive flux $\boldsymbol{F}_{\mathrm{C}}$, which can act both as a source or as a sink, depending on the temperature structure. The equation of energy balance in the stationary case is

$-E_{\mathrm{R}}+E_{\mathrm{H}}-\nabla \cdot \boldsymbol{F}_{\mathbf{C}}=0$.

For the purposes of this study, we assume a simple one-piece power-law radiative-loss function $E_{\mathrm{R}}$ (e.g., Kuin \& Martens 1982; Martens et al. 2000). For a Maxwellian plasma, it has the form

$E_{\mathrm{R}}(\chi, \sigma)=\chi n_{\mathrm{e}}^{2} T^{\sigma}$

where $\chi$ and $\sigma$ are two parameters characterizing the dependence of the radiative-loss function on temperature $T$. This radiativeloss function is similar to the one used by Rosner et al. (1978) and Serio et al. (1981) in deriving their respective scaling laws. The values of $\chi=10^{-31.81} \mathrm{Wm}^{3} \mathrm{~K}^{1 / 2}$ and $\sigma=-1 / 2$ (Kuin \& Martens 1982) are commonly assumed in deriving these scaling laws (e.g., Priest 1982). Here we relax these assumptions by treating both $\chi$ and $\sigma$ as parameters of the radiative-loss function.

The form of the volumetric heating function $E_{\mathrm{H}}$ is still unknown. It depends critically on the assumed physical heating mechanism(s). Here we make use of the parameterized form of the heating function introduced by Serio et al. (1981) and implemented by Aschwanden \& Schrijver (2002) as

$E_{\mathrm{H}}\left(s \geq s_{0}\right)=E_{\mathrm{H} 0} \exp \left(-\frac{s-s_{0}}{s_{\mathrm{H}}}\right)$.

This type of the heating function has two parameters: $E_{\mathrm{H} 0}$, the volumetric heating rate at the position $s_{0}$ (corresponding to the height $h_{0}$ ) of the chromospheric footpoint of the magnetic field line, and $s_{\mathrm{H}}$, the exponential heating scale-length measured along the field line. Obviously, the maximum of the heating function is localized at the footpoint $s_{0}$ and corresponds to the physical heating mechanisms that release heat energy mainly in the transition region and lower corona.

Thermal conductive flux $F_{\mathrm{C}}$ at position $s$ along a given magnetic field line is given by (Spitzer 1962)

$F_{\mathrm{C}}(s)=\kappa_{0} T^{5 / 2} \frac{\mathrm{d} T}{\mathrm{~d} s}$,

where $\kappa_{0} \approx 9.2 \times 10^{-12} \mathrm{Wm}^{-1} \mathrm{~K}^{-7 / 2}$ is the Spitzer thermal conduction coefficient. Since thermal conduction across the magnetic field can be neglected, this equation allows one to solve Eq. (7) as a one-dimensional problem.

Further assumptions about the thermal conduction profile along a magnetic field line are

$$
\begin{aligned}
& F_{\mathrm{C}}\left(s=s_{0}\right) \approx 0, \\
& F_{\mathrm{C}}\left(s=s_{1}\right)=0,
\end{aligned}
$$$$
F_{\mathrm{C}}\left(s_{0}<s<s_{1}\right) \neq 0 \text {, }
$$

which mean that the magnetic field line is thermally isolated and its temperature profile $T(s)$ is monotonic and has extrema only at both the footpoint and apex. These assumptions are necessary for integrating the temperature profile in both the temperature 
and spatial domains. In terms of a coronal loop, the assumptions given by Eqs. (11), (12) and (13) translates into a temperature maximum at the loop apex and a temperature minimum at the loop footpoint. The monotonical increase in temperature from footpoint to apex is ensured by the assumption in Eq. (13). The assumption in Eq. (11) means that the thermal conduction from the coronal loop into the chromosphere is negligible, i.e., the loop does not contribute to the heating of the underlying chromosphere. Equation (12) is the necessary, although not sufficient condition for loop symmetry. For the purposes of this paper, a geometrically symmetrical coronal loop of half-length $L=s_{1}$ heated equivalently at both footpoints will be assumed. The loop is treated as a slender fluxtube along the chosen magnetic field line, which defines the loop geometry, i.e., the loop is represented by a one-dimensional loop strand (Martens 2009). The effect of the varying loop cross-section is neglected. This question is discussed later in Sect. 3.5.

\subsection{Derivation of the scaling laws}

Equation (7) can be integrated to obtain analytical formulae describing coronal loops. The assumptions (11), (12), and (13) allow us to integrate from the footpoint $s_{0}$ to the loop apex $s_{1}=L$ in both the temperature and geometrical domains. The derivation here proceeds in a way analogous to Priest (1982).

First we rewrite the pressure stratification of the solar atmosphere given by Eq. (5) or Eq. (6) in terms of the loop coordinate $s$ and simplify it to the following form, which is equivalent to Eq. (9):

$p\left(s \geq s_{0}\right)=p_{0} \exp \left(-\frac{s-s_{0}}{s_{\mathrm{p}}}\right)$,

where $s_{\mathrm{p}}$ denotes the pressure scale length measured along the coronal loop. The discussion about the relation of $s_{\mathrm{p}}$ to the pressure scale height $\lambda_{\mathrm{p}}$ is given in Sect. 3.1.

In the one-dimensional form of Eq. (7), the divergence operator $\boldsymbol{\nabla}$. changes to $\mathrm{d} / \mathrm{d} s$. We multiply this equation by the factor $T^{5 / 2} \frac{\mathrm{d} T}{\mathrm{~d} s}$, then substitute into this equation the Eqs. (8), (9) and (10), and then substitute the electron density $n_{\mathrm{e}}$ by pressure according to Eqs. (1) and (14) to obtain

$$
\begin{aligned}
& \frac{1}{2} \kappa_{0} \frac{\mathrm{d}}{\mathrm{d} s}\left(T^{5 / 2} \frac{\mathrm{d} T}{\mathrm{~d} s}\right)^{2}= \\
& T^{5 / 2}\left(\frac{\chi p_{0}^{2} \mathrm{e}^{-2\left(s-s_{0}\right) / s_{\mathrm{p}}}}{q^{2} k_{\mathrm{B}}^{2}} T^{\sigma-2}-E_{\mathrm{H} 0} \mathrm{e}^{-\left(s-s_{0}\right) / s_{\mathrm{H}}}\right) \frac{\mathrm{d} T}{\mathrm{~d} s} .
\end{aligned}
$$

Direct integration of this equation over the temperature range from the apex (coronal) temperature $T_{1}$ to the lower transitionregion temperature $T_{0}$ is impossible, since the pressure scalelength $s_{\mathrm{p}}$ is directly dependent on the temperature and height profiles, i.e., $s_{\mathrm{p}}=s_{\mathrm{p}}(T(s), h(s)$ ) (cf. Eq. (35)) and $\sigma$ is not in general a natural number. However, the mean-value theorem (Appendix A) can be used to obtain the following expression

$$
\begin{aligned}
0= & \frac{\chi p_{0}^{2} \mathrm{e}^{-\beta\left(L-s_{0}\right) / s_{\mathrm{p}}}}{q^{2} k_{\mathrm{B}}^{2}} \frac{T_{0}^{\sigma+3 / 2}-T_{1}^{\sigma+3 / 2}}{\sigma+3 / 2} \\
& -\frac{2}{7} E_{\mathrm{H} 0}\left(T_{0}^{7 / 2}-T_{1}^{7 / 2}\right) \mathrm{e}^{-\gamma\left(L-s_{0}\right) / s_{\mathrm{H}}},
\end{aligned}
$$

where $\beta$ and $\gamma$ are constants to be determined. The left-hand side of Eq. (16) equals zero because of the assumptions (11) and (12).

In the case of a typical coronal loop, $T_{1} \gg T_{0}$. If $\sigma>-3 / 2$, then all terms containing $T_{0}$ can be neglected. In the case of $\sigma<-3 / 2$, the (coronal) loop apex temperature $T_{1}$ would be strongly coupled to the temperature of the chromosphere. In the following, $\sigma>-3 / 2$ will always be assumed. Neglecting $T_{0}$ immediately results in the following expression for the base pressure

$p_{0}^{2}=\frac{3+2 \sigma}{7} \frac{q^{2} k_{\mathrm{B}}^{2}}{\chi} E_{\mathrm{H} 0} T_{1}^{2-\sigma} \mathrm{e}^{\beta\left(L-s_{0}\right) / s_{\mathrm{p}}} \mathrm{e}^{-\gamma\left(L-s_{0}\right) / s_{\mathrm{H}}}$.

To obtain a second relation describing the coronal loop, Eq. (15) is integrated again from $T_{1}$ to a temperature $T^{\prime}, T_{0}<T^{\prime} \leq T_{1}$, i.e., as the function of the integral boundary. Substituting for $E_{\mathrm{H} 0}$ from Eq. (17), using the mean-value theorem and rearranging the equation leads to

$$
\begin{gathered}
T^{\prime 5}\left(\frac{\mathrm{d} T^{\prime}}{\mathrm{d} s}\right)^{2}=\frac{4 \chi p_{0}^{2}}{q^{2} k_{\mathrm{B}}^{2} K_{0}(3+2 \sigma)} \mathrm{e}^{-\beta^{\prime}\left(L-s_{0}\right) / s_{\mathrm{p}}}\left(T^{\sigma+3 / 2}-T_{1}^{\sigma+3 / 2}-\right. \\
\left.\left(T_{1}^{\sigma-2} T^{\prime 7 / 2}-T_{1}^{\sigma+3 / 2}\right) \mathrm{e}^{-\left(\beta-\beta^{\prime}\right)\left(L-s_{0}\right) / s_{\mathrm{p}}} \mathrm{e}^{\left(\gamma-\gamma^{\prime}\right)\left(L-s_{0}\right) / s_{\mathrm{H}}}\right)
\end{gathered}
$$

where $\beta^{\prime}$ and $\gamma^{\prime}$ are a pair of coefficients that are in general functions of $T^{\prime}$. In the following, the expression

$P=\mathrm{e}^{-\left(\beta-\beta^{\prime}\right)\left(L-s_{0}\right) / s_{\mathrm{p}}} \mathrm{e}^{\left(\gamma-\gamma^{\prime}\right)\left(L-s_{0}\right) / s_{\mathrm{H}}}$

will be used. Dividing the Eq. (18) by $T^{\prime \sigma+3 / 2}$, extracting the square-root, and integrating it again from $T_{0}$ to $T_{1}$ in the temperature domain and from $s_{0}$ to $L$ in the corresponding spatial domain will, after substituting $x=T^{\prime} / T_{1}$, lead to

$$
\begin{aligned}
T_{1}^{1+\mu} \int_{T_{0} / T_{1}}^{1} \frac{x^{\mu} \mathrm{d} x}{\sqrt{1-(1-P) x^{\theta}-P x^{\nu}}}= \\
\left(\frac{2}{3+2 \sigma} \frac{2 \chi}{q^{2} k_{\mathrm{B}}^{2} \kappa_{0}}\right)^{1 / 2} \mathrm{e}^{-\beta^{\prime \prime}\left(L-s_{0}\right) / 2 s_{\mathrm{p}}} p_{0}\left(L-s_{0}\right),
\end{aligned}
$$

where the expressions $\mu=(7-2 \sigma) / 4, v=2-\sigma$, and $\theta=-\sigma-3 / 2$ have been used. For $\sigma>-3 / 2, \mu, v>0$ and $\theta<0$. The $\beta^{\prime \prime}$ represents here the mean value of $\beta^{\prime}$ when integrated over $s$ in the entire coronal portion of the loop. Similarly, $\gamma^{\prime \prime}$ stands for the mean value of $\gamma^{\prime}$. This equation is analogous to Eq. (6) of Kuin $\&$ Martens (1982), which relates the radiative-loss-weighted values of temperature $T_{i}$ and density $n_{i}$ in a uniformly heated loop with homogeneous pressure. In our case, Eq. (20) relates the apex temperature $T_{1}$ to the base pressure $p_{0}$ when assuming a pressure-stratified loop and non-uniform heating.

The value of $T_{0}$ with respect to $T_{1}$ can again be neglected. The integral function

$I(\sigma, P)=\int_{0}^{1} \frac{x^{\mu} \mathrm{d} x}{\sqrt{1-(1-P) x^{\theta}-P x^{\nu}}}$,

which exists only if $P \geq 1$, is bounded and easily calculated numerically, despite the divergence of the sub-integral function for $x \rightarrow 1$. We note that $P$ is in general a function of $x$, since $\beta^{\prime}$ and $\gamma^{\prime}$ are functions of $T^{\prime}$. However, since little assumption about the temperature profile $T(s)$ has been made, there is no information about the function $P(x)$. Thus, $P$ is assumed to be constant for to some values $\beta^{\prime \prime \prime}$ and $\gamma^{\prime \prime \prime}$ :

$P=\mathrm{e}^{-\left(\beta-\beta^{\prime \prime \prime}\right) L_{0} / s_{\mathrm{p}}} \mathrm{e}^{\left(\gamma-\gamma^{\prime \prime \prime}\right) L_{0} / s_{\mathrm{H}}}$.

The condition $P \geq 1$ results in formal bounding for the heating scale-height:

$s_{\mathrm{H}} \leq \frac{\gamma-\gamma^{\prime \prime \prime}}{\beta-\beta^{\prime \prime \prime}} s_{\mathrm{p}}$. 

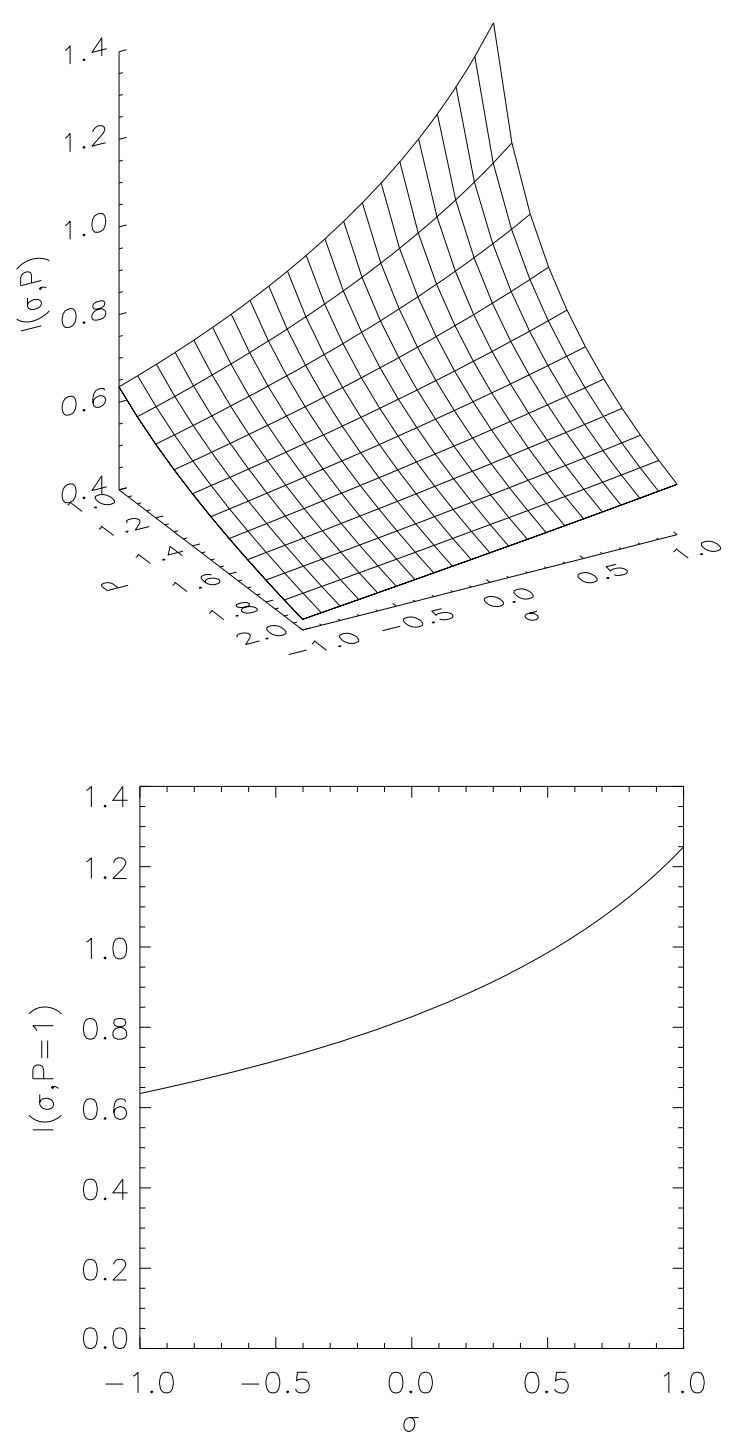

Fig. 1. Top: the $I(\sigma, P)$ function, plotted for $-1 \leq \sigma \leq 1$ and $1 \leq P \leq 2$. Bottom: the $I(\sigma, P=1)$ function plotted for the same range in $\sigma$.

The shape of the $I(\sigma, P)$ function is depicted in Fig. 1. The special case of $I(\sigma=-1 / 2, P=1) \approx 0.72$, corresponding to the scaling laws of Rosner et al. (1978), was already derived by Priest (1982, p. 237-8).

If the set of variables $\left(L_{0}, E_{\mathrm{H} 0}, s_{\mathrm{H}}\right)$, where $L_{0}=L-s_{0}$ is considered to be independent and $T_{1}$ and $p_{0}$ to be dependent variables, the scaling laws (17) and (20) can be easily rewritten to obtain the following expressions for $T_{1}$ and $p_{0}$ :

$$
\begin{aligned}
T_{1}= & \left(\frac{4}{7 \kappa_{0}}\right)^{2 / 7} I(\sigma, P)^{-4 / 7} E_{\mathrm{H} 0}^{2 / 7}\left(L_{0}\right)^{4 / 7} \exp \left(-\frac{2}{7} \gamma \frac{L_{0}}{s_{\mathrm{H}}}\right) \\
& . \exp \left(\frac{2}{7}\left(\beta-\beta^{\prime \prime}\right) \frac{L_{0}}{s_{\mathrm{p}}}\right) \\
p_{0}^{2}= & \frac{3+2 \sigma}{7} \frac{q^{2} k_{\mathrm{B}}^{2}}{\chi}\left(\frac{4}{7 \kappa_{0}}\right)^{(4-2 \sigma) / 7} I(\sigma, P)^{(4 \sigma-8) / 7} E_{\mathrm{H} 0}^{(11-2 \sigma) / 7} \\
& .\left(L_{0}\right)^{(8-4 \sigma) / 7} \cdot \exp \left(-\gamma \frac{11-2 \sigma}{7} \frac{L_{0}}{s_{\mathrm{H}}}\right) \\
& . \exp \left(+\beta \frac{11-2 \sigma}{7} \frac{L_{0}}{s_{\mathrm{p}}}\right) \exp \left(-\beta^{\prime \prime} \frac{4-2 \sigma}{7} \frac{L_{0}}{s_{\mathrm{p}}}\right) .
\end{aligned}
$$

Table 1. Best-fit model coefficients in scaling laws expressions (32) and (31).

\begin{tabular}{lllllll}
\hline \hline$T_{1}[\mathrm{MK}]$ & $P$ & $P^{\prime}$ & $\beta_{1}$ & $\beta_{2}$ & $\gamma_{1}$ & $\gamma_{2}$ \\
\hline 1 & 1.046 & 0.958 & -0.010 & -0.136 & -0.062 & 0.709 \\
3 & 1.007 & 0.958 & +0.005 & -0.469 & -0.093 & 0.829 \\
5 & 1.005 & 0.949 & +0.022 & -0.721 & -0.095 & 0.861 \\
10 & 1.069 & 0.762 & +0.025 & -0.808 & -0.068 & 0.778 \\
\hline
\end{tabular}

Alternatively, the scaling laws can be written for the choice of $\left(L_{0}, s_{\mathrm{H}}, T_{1}\right)$ as independent and $\left(p_{0}, E_{\mathrm{H} 0}\right)$ as dependent variables, i.e., in the form compatible with Eqs. (29) and (30) of Aschwanden \& Schrijver (2002). In this form, the scaling laws become

$$
\begin{aligned}
p_{0}\left(L_{0}, s_{\mathrm{H}}, T_{1}\right) & =L_{0}^{-1} T_{1}^{(11-2 \sigma) / 4} S_{1}^{-3}, \\
E_{\mathrm{H} 0}\left(L_{0}, s_{\mathrm{H}}, T_{1}\right) & =L_{0}^{-2} T_{1}^{7 / 2} S_{2},
\end{aligned}
$$

while the scaling law expressions $S_{1}$ and $S_{2}$ in our version (DDKK) take the form

$$
\begin{aligned}
& S_{1}^{\text {DDKK }}=\left(\frac{3+2 \sigma}{7} \frac{q^{2} k_{\mathrm{B}}^{2}}{\chi} \frac{7 \kappa_{0}}{4} I(\sigma, P)^{2} \mathrm{e}^{\beta^{\prime \prime} L_{0} / s_{\mathrm{p}}}\right)^{-1 / 6}, \\
& S_{2}^{\text {DDKK }}=I(\sigma, P)^{2} \frac{7 \kappa_{0}}{4} \mathrm{e}^{-\left(\beta-\beta^{\prime \prime}\right) L_{0} / s_{\mathrm{p}}} \mathrm{e}^{\gamma L_{0} / s_{\mathrm{H}}} .
\end{aligned}
$$

From Eq. (26), it is clear that the dependency of base pressure $p_{0}$ on the apex temperature $T_{1}$ is modified by the presence of the radiative-loss function parameter $\sigma$. For $\sigma=-1 / 2$, the temperature exponent is equal to 3 . The dependency of $p_{0}$ on $S_{1}$ of the inverse third-power is retained, although the $S_{1}$ itself depends on $\sigma$.

Equation (22) can be used to obtain

$\mathrm{e}^{\beta^{\prime \prime} L_{0} / s_{\mathrm{p}}}=\mathrm{e}^{\left(\beta^{\prime \prime \prime} L_{0} / s_{\mathrm{p}}\right)\left(\beta^{\prime \prime} / \beta^{\prime \prime \prime}\right)}=P^{\prime} \mathrm{e}^{\beta \beta^{\prime \prime} L_{0} / \beta^{\prime \prime \prime} s_{\mathrm{p}}} \mathrm{e}^{\left(\gamma^{\prime \prime \prime}-\gamma\right) \beta^{\prime \prime} L_{0} / \beta^{\prime \prime \prime} s_{\mathrm{H}}}$,

where $P^{\prime}=P^{\beta^{\prime \prime}} \mid \beta^{\prime \prime \prime}$. Defining $\beta_{1}=-\beta \beta^{\prime \prime} / 6 \beta^{\prime \prime \prime}, \gamma_{1}=(\gamma-$ $\left.\gamma^{\prime \prime \prime}\right) \beta^{\prime \prime} / 6 \beta^{\prime \prime \prime}, \gamma_{2}=\gamma$, and $\beta_{2}=\beta^{\prime \prime}-\beta$ gives

$$
\begin{aligned}
& S_{1}^{\text {DDKK }}=\left(\frac{3+2 \sigma}{7} \frac{q^{2} k_{\mathrm{B}}^{2}}{\chi} \frac{7 \kappa_{0}}{4} I(\sigma, P)^{2} P^{\prime}\right)^{-1 / 6} \mathrm{e}^{\beta_{1} L_{0} / s_{\mathrm{p}}} \mathrm{e}^{\gamma_{1} L_{0} / s_{\mathrm{H}}} \\
& S_{2}^{\text {DDKK }}=I(\sigma, P)^{2} \frac{7 \kappa_{0}}{4} \mathrm{e}^{\beta_{2} L_{0} / s_{\mathrm{p}}} \mathrm{e}^{\gamma_{2} L_{0} / s_{\mathrm{H}}}
\end{aligned}
$$

This form of the scaling laws for coronal loops is only a formal one, and for the completion one needs to determine the values of $P, P^{\prime}, \beta_{1}, \beta_{2}, \gamma_{1}$, and $\gamma_{2}$. Unfortunately, without directly assuming the function $T(s)$, their values cannot be determined analytically. Aschwanden \& Schrijver (2002) found good analytical approximations of the function $T(s)$ that depend on the geometrical properties of the loop, i.e., values of $L$ and $s_{\mathrm{H}}$, and also the apex temperature $T_{1}$ (cf. their Eqs. (19)-(21) and Table 1). This means that the values of $P, P^{\prime}, \beta_{1}, \beta_{2}, \gamma_{1}$, and $\gamma_{2}$ can depend on the loop parameters $L, s_{\mathrm{H}}$, and $T_{1}$, i.e., they can change from loop to loop.

At this point an adequate approximation is required. The simplest assumption that can be made is that these parameters are constants for the entire ranges of $L$ and $s_{\mathrm{H}}$, while they (obviously) must change with the apex temperature $T_{1}$. In other words, we assume that the temperature profile does not change much with $L$ and $s_{\mathrm{H}}$. For this assumption, one can expect that the value of $P$ will be close to unity, since $P$ must approach unity for low $L / s_{\mathrm{H}}$ values and shorter $L$, where the scaling laws of Rosner et al. (1978) hold. To find the values of $P, P^{\prime}, \beta_{1}, \beta_{2}, \gamma_{1}$, and 

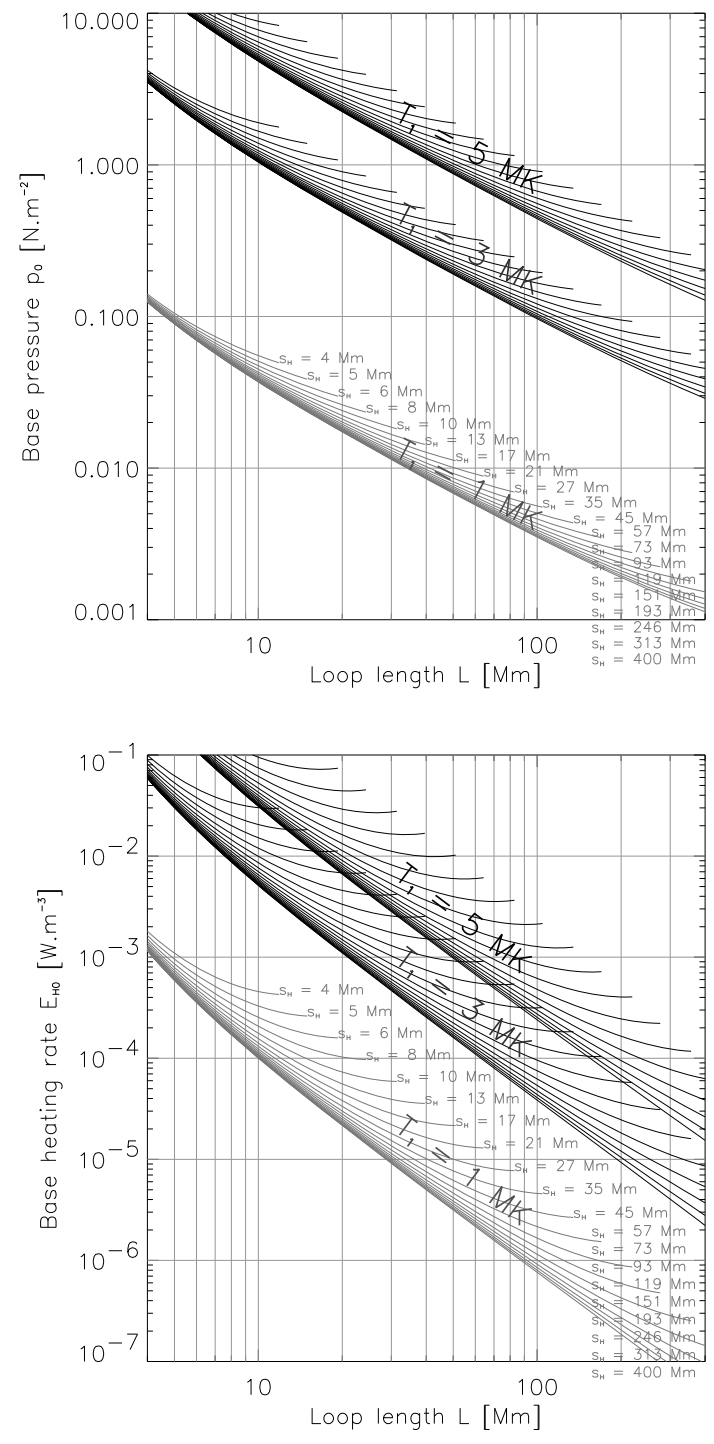

Fig. 2. Scaling law for the base pressure $p_{0}$ (Eqs. (26) and (31)) and base heating rate (Eqs. (27) and (32)) as a functions of $L, s_{\mathrm{H}}$, and $T_{1}$, for $\sigma=-1 / 2$, and $\chi=10^{-31.88} \mathrm{Wm}^{3} \mathrm{~K}^{1 / 2}$. Lines corresponding to a given $s_{\mathrm{H}}$ are terminated at points where $L / s_{\mathrm{H}}=3$.

$\gamma_{2}$, a numerical method for solving Eq. (7) with the appropriate boundary conditions must be invoked. The values of $P, P^{\prime}, \beta_{1}$, $\beta_{2}, \gamma_{1}$, and $\gamma_{2}$ can then be determined by direct fitting of the scaling laws (26) and (27) to the results of numerical simulations, for the assumption that $s_{\mathrm{p}}$ is the pressure scale-length corresponding to the apex temperature $T_{1}$.

To do this, we used the numeric code developed by Aschwanden \& Schrijver (2002), which is part of the hydro package of the SolarSoft environment (Freeland \& Handy 1998), running as a layer on top of the Interactive Data Language (IDL). The code enables one to choose from several implemented radiative-loss functions and then calculate solutions to Eq. (7). The chosen values of $T_{0}$ and $T_{1}$ together with Eqs. (11) and (12) represent the boundary conditions of a given loop. A fixed choice of $T_{1}$ removes the difficulty of $s_{\mathrm{p}}$ being a function of apex temperature. The value of $s_{0}$ is determined by the height of the chromosphere together with the assumed loop geometry. We used the values of $h_{0}=1.3 \mathrm{Mm}$ and $T_{0}=2 \times 10^{4} \mathrm{~K}$ for the upper chromosphere, and $L$ and $s_{\mathrm{H}}$ acted as independent variables.
The code returns the values of $E_{\mathrm{H} 0}$ and $p_{0}$. Assuming a semicircular loop geometry and executing the code multiple times for different choices of $L$ and $s_{\mathrm{H}}$, we constructed a numerical grid of $E_{\mathrm{H} 0}$ and $p_{0}$ as functions of $L$ and $s_{\mathrm{H}}$. The values of $P$, $P^{\prime}, \beta_{1}, \beta_{2}, \gamma_{1}$, and $\gamma_{2}$ were then determined by the LevenbergMarquardt method (Marquardt 1963), which minimizes the difference between the grid of numerical models and the scaling laws given by Eqs. (26) and (27) together with Eqs. (31) and (32). We indicate the parameter values for the choice of $\sigma=-1 / 2, \chi=10^{-31.88} \mathrm{Wm}^{3} \mathrm{~K}^{1 / 2}$ (Kuin \& Martens 1982), and $4 \mathrm{Mm} \leq L, s_{\mathrm{H}} \leq 400 \mathrm{Mm}$, which is the same parameter space used by Aschwanden \& Schrijver (2002). Since the computed numerical values of $p_{0}$ and $E_{\mathrm{H} 0}$ span two and four orders of magnitude, respectively, the minimization was performed in logarithmic space. The results are summarized in Table 1.

For $L / s_{\mathrm{H}}>3$, the points in parameter space were excluded from the fitting procedure, because the numerical code often failed to converge here. The fitting accuracy of scaling laws is $\leq 40 \%$ for $E_{\mathrm{H} 0}$ and $\leq 15 \%$ for $p_{0}$. The accuracy is higher for lower $L / s_{\mathrm{H}}$ values and rapidly decreased towards the $L / s_{\mathrm{H}} \sim 3$ boundary. This is because for higher $L / s_{\mathrm{H}}$ values, the function $T(s)$ changes rapidly (cf. Fig. 9 of Aschwanden \& Schrijver 2002). The accuracy of the numerical solutions is less than $2 \%$.

The resulting dependency of $p_{0}$ and $E_{\mathrm{H} 0}$ on $L, s_{\mathrm{H}}$, and $T_{1}$ is plotted in Fig. 2. In Fig. 3, the scaling laws are compared with numerical solutions and previously derived scaling laws of Rosner et al. (1978), Serio et al. (1981) and Aschwanden \& Schrijver (2002). These plots are compiled in the same way as Figs. 4-6 of Aschwanden \& Schrijver (2002) to facilitate direct visual comparison.

Slightly higher accuracy can be achieved by relaxing the assumption that $\beta_{1}, \beta_{2}, \gamma_{1}$, and $\gamma_{2}$ are constants. If these parameters are allowed to vary slightly with $L$ and $s_{\mathrm{H}}$, the scaling law expressions in Eqs. (31) and (32) can be expanded into Taylor series. Neglecting terms higher than linear leads to

$$
\begin{aligned}
S_{1}^{\mathrm{DDKK}} & =\left(\frac{3+2 \sigma}{7} \frac{q^{2} k_{\mathrm{B}}^{2}}{\chi} \frac{7 \kappa_{0}}{4} I(\sigma, P)^{2} P^{\prime}\right)^{-1 / 6} . \\
& \cdot \mathrm{e}^{\beta_{1}^{(0)} L_{0} / s_{\mathrm{p}}} \mathrm{e}^{\gamma_{1}^{(0)} L_{0} / s_{\mathrm{H}}}\left(1+\frac{L_{0}}{s_{\mathrm{p}}} \Delta \beta_{1}+\frac{L_{0}}{s_{\mathrm{H}}} \Delta \gamma_{1}\right),
\end{aligned}
$$

$$
\begin{aligned}
S_{2}^{\mathrm{DDKK}} & =I(\sigma, P)^{2} \frac{7 \kappa_{0}}{4} . \\
& \cdot \mathrm{e}^{\beta_{2}^{(0)} L_{0} / s_{\mathrm{p}}} \mathrm{e}^{\gamma_{2}^{(0)} L_{0} / s_{\mathrm{H}}}\left(1+\frac{L_{0}}{s_{\mathrm{p}}} \Delta \beta_{2}+\frac{L_{0}}{s_{\mathrm{H}}} \Delta \gamma_{2}\right),
\end{aligned}
$$

where the expressions $\Delta \beta_{1,2}=\beta_{1,2}-\beta_{1,2}^{(0)}$, and $\Delta \gamma_{1,2}=\gamma_{1,2}-$ $\gamma_{1,2}^{(0)}$ have been used. Once again, we assume that $P, P^{\prime}, \beta_{1,2}^{0}$, $\gamma_{1,2}^{(0)}, \Delta \beta_{1,2}$, and $\Delta \gamma_{1,2}$ are constants to be determined by fitting the results of numerical simulations. The fitting accuracy is $\leq 25-35 \%$ for $E_{\mathrm{H} 0}$ and $\leq 7-9 \%$ for $p_{0}$. We note that the scaling law expressions (33) and (34) differ from the empirical scaling law expressions of Aschwanden \& Schrijver (2002, Eqs. (35) and (36) therein). The correction terms in our scaling law expressions (33) and (34) are strongly dependent on $L_{0}$ and $s_{\mathrm{H}}$. The correction terms in the empirical scaling law expressions of Aschwanden \& Schrijver (2002), which contain a weaker dependency on $L_{0}$ and $s_{\mathrm{H}}$, allow for higher fitting accuracy. This accuracy is reported to be $\leq 5 \%$ by these authors. However, inspection of their Fig. 4 bottom left panel suggests that the accuracy is closer to $\leq 10 \%$ for $E_{\mathrm{H} 0}$. This accuracy could also be 

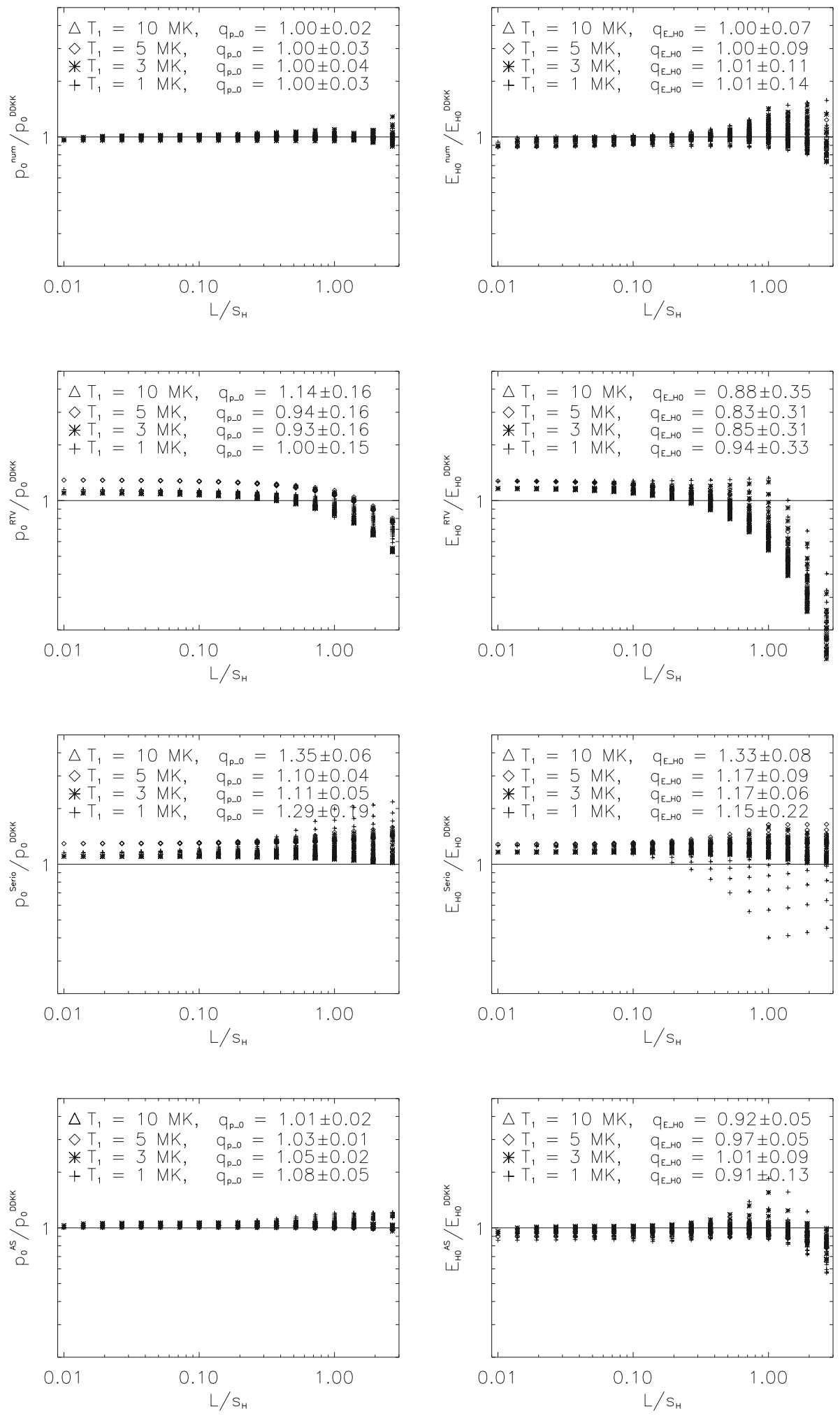

Fig. 3. Comparison of "DDKK" scaling laws (Eqs. (26), (27) together with Eqs. (31) and (32)) with the numerical solutions (top row) and previously derived scaling laws of Rosner et al. (1978, second row), Serio et al. (1981, third row), and Aschwanden \& Schrijver (2002, bottom row). Average ratios and standard deviations are given in each figure for each temperature separately.

achieved in our case, if we would fit their scaling law expressions (35) and (36) with the results of numerical simulations. We note that the difference between the numerical simulations of Aschwanden \& Schrijver (2002) and the numerical simulations computed here is in the type of radiative-loss function addopted. While they used the radiative-loss function computed by Tucker \& Koren (1971), we use the one-piece power-law approximation of this function given by Kuin \& Martens (1982) (cf. Fig. 7 in Aschwanden \& Schrijver 2002). We conclude that the correction terms in scaling law expressions containing terms linear in $L_{0} / s_{\mathrm{p}}$, and $L_{0} / s_{\mathrm{H}}$, as suggested by Aschwanden \& Schrijver (2002), are more appropriate to reproducing the numerical simulations, although they are merely empirical, since they have little physical justification. However, our derivation justifies the $d_{0}$ and $e_{0}$ parameters of Aschwanden \& Schrijver (2002) 

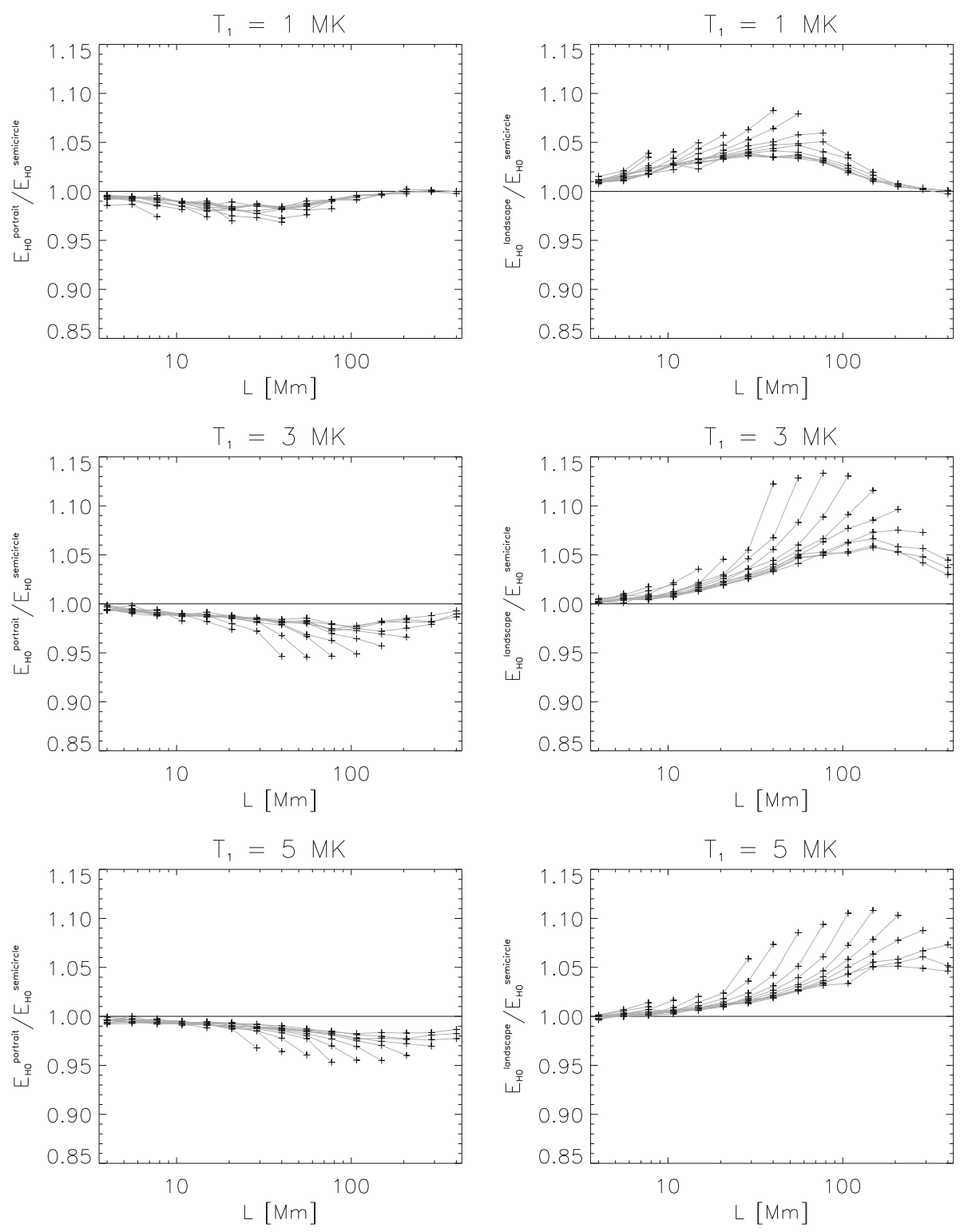

Fig. 4. Comparison of the numerical results for the base pressure $E_{\mathrm{H} 0}$ for elliptical and circular loop geometries. Left column: elliptical, portrait-oriented loops vs. semicircular loops. Right column: elliptical, landscape-oriented loops vs. semicircular loops. Computed numerical values are denoted by the "+" sign. Different lines in plots stands for different values of heating scale length $s_{\mathrm{H}}$. From left to right: $s_{\mathrm{H}}=4 \mathrm{Mm}, 5.5 \mathrm{Mm}, 7.7 \mathrm{Mm}$, $10.7 \mathrm{Mm}, 14.9 \mathrm{Mm}, 20.7 \mathrm{Mm}, 28.7 \mathrm{Mm}$, $40 \mathrm{Mm}, 55.5 \mathrm{Mm}, 77.2 \mathrm{Mm}, 107.3 \mathrm{Mm}$, 149.1 Mm, 207.2 Mm, 287.9 $\mathrm{Mm}$ and $400 \mathrm{Mm}$. Maximum plotted $L / s_{\mathrm{H}}$ values are approximately 1.9 .

in terms of the parameter $P^{\prime}$ and the integral function $I(\sigma, P)$ in Eq. (21).

\section{Discussion}

\subsection{Hydrostatic scale-height}

We note that the pressure scale-length $s_{\mathrm{p}}$ is not equal to the pressure scale-height $\lambda_{\mathrm{p}}$. The pressure scale-length $s_{\mathrm{p}}$ is defined as the distance along the loop between points of pressure $p\left(s_{0}\right)=p_{0}$ and $p\left(s_{\mathrm{p}}+s_{0}\right)=p_{0} / \mathrm{e}$. In contrast, pressure scaleheight $\lambda_{\mathrm{p}}$ is the vertical distance between the points $s_{0}$, corresponding to height $h_{0}$, and $s_{\mathrm{p}}+s_{0}$, corresponding to height $\lambda_{\mathrm{p}}+h_{0}$. Since for a given loop geometry, the relation $h=h(s)$ and the inverse relation $s=s(h)$ is known, using the Eqs. (6) and (14), we can write approximately

$s_{\mathrm{p}} \approx \frac{s-s_{0}}{h-h_{0}} \lambda_{\mathrm{p}}$.

With the knowledge of the loop apex height $h_{1}$, we can estimate $s_{\mathrm{p}}$ to be

$s_{\mathrm{p}} \approx \frac{L-s_{0}}{h_{1}-h_{0}} \lambda_{\mathrm{p}}$.
This equation was used in constructing the grid of numerical models in Sect. 2.2 for the assumption of semi-circular loop geometry.

\subsection{Elliptical loops - a case study}

In this section we perform a case study of the importance of different loop geometries on the resulting distributions of base pressure $p_{0}$ and volumetric heating rate $E_{\mathrm{H} 0}$ as functions of ( $L$, $\left.s_{\mathrm{H}}, T_{1}\right)$. The effects of varying loop geometry on hydrostatic loop solutions has not been studied previously the literature. Any possible effects on the resulting emission models were neglected (e.g., Schrijver et al. 2004).

We computed a grid of numerical models identical to those in Sect. 2.2, but for loops of semi-elliptical shape. The semimajor to semi-minor axis ratio of these loops was chosen to equal 2 , which corresponds to an eccentricity of $\sqrt{3} / 2$. In the first case, the semi-elliptical loops are oriented in portrait form, i.e., the photospheric footpoint baseline of the semi-elliptical loop is about a factor of 1.541 shorter than the photospheric footpoint baseline of the semi-circular loop with the same halflength $L$, while the apex is located 1.298-times higher. In the 

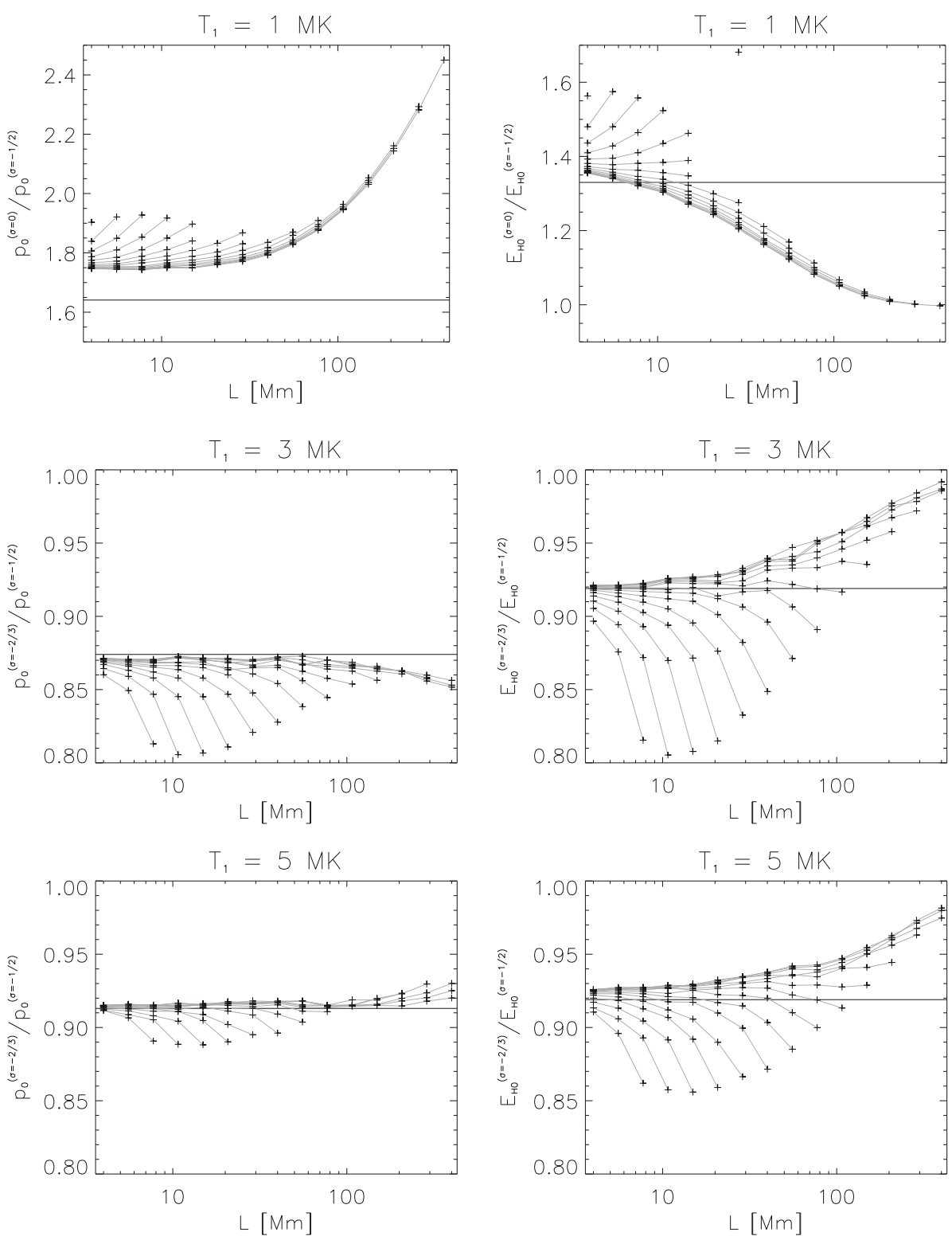

Fig. 5. Comparison of the numerical results for the base pressure $p_{0}$ and base heating rate $E_{\mathrm{H} 0}$ for the two radiative-loss functions $E_{\mathrm{R} 1}=\chi_{1} n_{\mathrm{e}}^{2} T^{\sigma_{1}}$ and $E_{\mathrm{R} 0}=\chi_{0} n_{\mathrm{e}}^{2} T^{\sigma_{0}}$ with $\chi_{1}=$ $10^{-34.94} \mathrm{Wm}^{3}, \sigma_{1}=0, \chi_{0}=10^{-31.81} \mathrm{Wm}^{3} \mathrm{~K}^{1 / 2}$ and $\sigma_{0}=-1 / 2$. The black lines in both pictures correspond to the estimated ratio values. Computed numerical values are denoted by the "+" sign. Gray lines connect points with constant values of the heating scale length $s_{\mathrm{H}}$. From left to right: $s_{\mathrm{H}}=4 \mathrm{Mm}, 5.5 \mathrm{Mm}, 7.7 \mathrm{Mm}$, $10.7 \mathrm{Mm}, 14.9 \mathrm{Mm}, 20.7 \mathrm{Mm}, 28.7 \mathrm{Mm}$, $40 \mathrm{Mm}, 55.5 \mathrm{Mm}, 77.2 \mathrm{Mm}, 107.3 \mathrm{Mm}$, 149.1 Mm, 207.2 Mm, 287.9 $\mathrm{Mm}$ and $400 \mathrm{Mm}$. Maximum plotted $L / s_{\mathrm{H}}$ values are approximately 1.0. Higher values are not plotted due to abundance of numerical errors for the case of $E_{\mathrm{R} 1}$.
Fig. 6. Same as in Fig. 5, but for the two radiative-loss functions $E_{\mathrm{R} 2}=\chi_{2} n_{\mathrm{e}}^{2} T^{\sigma_{2}}$ and $E_{\mathrm{R} 0}=\chi_{0} n_{\mathrm{e}}^{2} T^{\sigma_{0}}$ with $\chi_{2}=10^{-30.73} \mathrm{Wm}^{3} \mathrm{~K}^{2 / 3}$, $\sigma_{2}=-2 / 3$. Maximum plotted $L / s_{\mathrm{H}}$ values are about 1.9 . second studied case, the semi-elliptical loops are oriented landscape, i.e., the photospheric footpoint baseline of a such semielliptical loop is 1.298-times longer than the baseline of the semi-circular loop with the same half-length, while the apex is located $1 / 1.541 \approx 0.649$-times lower.

For the purpose of constructing the grid of numerical models, we modified the hydro_numeric.pro and hydro_numeric_func.pro routines of the hydro package by changing the employed $h=h(s)$ function, which is for semi-elliptical loops given by the elliptic integral of the second kind, together with the component of gravitational force acting along the loop. We computed the grid of numerical models for four apex temperatures $T_{1}=1,3,5$, and $10 \mathrm{MK}$. Unfortunately, we found that the numerical grid for the latest apex temperature was impossible to construct, because we encountered serious problems related to the spatial distribution of points $s$ along loops. The problem appears for the entire range of recommended spatial distribution parameters (Aschwanden, private communication) and is left unresolved. However, computations for the first three apex temperatures were not affected by this problem. The results for the base heating rate $E_{\mathrm{H} 0}$ for these apex temperatures are displayed in Fig. 4. Taking into account that the error in the numerical simulations is less than $2 \%$, we conclude that the effect of elliptical loop geometry is $\leq 13 \%$ for the base heating rate $E_{\mathrm{H} 0}$ for loops with $L / s_{\mathrm{H}} \leq 2$. We note that because of Eq. (17), the relative changes in the base pressure $p_{0}$ are expected to scale as the square root of the changes in the base heating rate $E_{\mathrm{H} 0}$. Thus, the semi-elliptical loop geometry causes less than a $\sim 6 \%$ difference in the base pressure $p_{0}$.

The total changes $\Delta E_{\mathrm{R}}$ in the radiative output of a loop due to the different loop geometry then scale as

$\Delta E_{\mathrm{R}} \sim \Delta E_{\mathrm{H} 0}+\Delta\left(p_{0}^{2}\right) \sim 2 \Delta E_{\mathrm{H} 0}$.

We are unable to study the effects of elliptical loop geometry for higher values of the $L / s_{\mathrm{H}}$ ratio, since the code fails to converge for $L / s_{\mathrm{H}} \sim 3$ more often in the case of semi-elliptical loops than for semi-circular loops. However, the overall trend is evident from Fig. 4 . With the increasing $L / s_{\mathrm{H}}$ ratio, the differences related to geometry start to rise, reaching maxima for 
$s_{\mathrm{H}} \sim 30 \mathrm{Mm}$ for $T_{1}=1 \mathrm{MK}$. The maxima move progressively to higher $s_{\mathrm{H}}$ values for increasing apex temperature. The effect is also more pronounced for landscape-oriented loops than for portrait-oriented loops.

\subsection{Effect of radiative-loss function}

We now study the effects of different radiative-loss functions on the density and temperature structure of the coronal loops. On the one hand, Eq. (26) together with Eq. (31) show the explicit dependence of the base pressure on the parameters $\chi$ and $\sigma$ of the radiative-loss function (8). If we assume two different radiativeloss functions, $E_{\mathrm{R} 1}=\chi_{1} n_{\mathrm{e}}^{2} T^{\sigma_{1}}$ and $E_{\mathrm{R} 2}=\chi_{2} n_{\mathrm{e}}^{2} T^{\sigma_{2}}$, we obtain the following expression for the ratio of the resulting base pressures

$\frac{p_{0}^{(1)}}{p_{0}^{(2)}}=T_{1}^{-\frac{1}{2}\left(\sigma_{1}-\sigma_{2}\right)} \frac{I\left(\sigma_{1}, P_{1}\right)}{I\left(\sigma_{2}, P_{2}\right)} \sqrt{\frac{\chi_{2}}{\chi_{1}}} \sqrt{\frac{3+2 \sigma_{1}}{3+2 \sigma_{2}}}$,

where $P_{1}$ and $P_{2}$ can in general be different values for different radiative-loss functions. Similarly, the ratio of the resulting base heating rates $E_{\mathrm{H} 0}^{(1)} / E_{\mathrm{H} 0}^{(2)}$ is given by the expression $I\left(\sigma_{1}, P_{1}\right)^{2} / I\left(\sigma_{2}, P_{2}\right)^{2}$ (Eqs. (27) and (32)).

On the other hand, Eqs. (27) and (32) suggest that the volumetric base heating rate $E_{\mathrm{H} 0}$ does not depend on $\chi$, and depends only weakly on $\sigma$ through the $I(\sigma, P)$ function. This result is confusing, since changes in total radiative output could in general require adjustments to the total energy input so that the assumed energy balance given by Eq. (7) is kept throughout the loop, even if the heating scale-length remains fixed, i.e., the volumetric heating rate $E_{\mathrm{H} 0}$ could in general be dependent on $\chi$ and $\sigma$.

To study this in more detail, we constructed a grid of numerical models using the radiative-loss functions $E_{\mathrm{R} 1}$ and $E_{\mathrm{R} 2}$ with $\chi_{1}=10^{-34.94} \mathrm{Wm}^{3}, \sigma_{1}=0$ and $\chi_{2}=10^{-30.73} \mathrm{Wm}^{3} \mathrm{~K}^{2 / 3}$, $\sigma_{2}=-2 / 3$, which are the last two parts of the Rosner et al. (1978, Appendix A therein) analytical fit to the radiative-loss function of Raymond \& Smith (1977). The first approximation is valid within the temperature range $10^{5.75} \mathrm{~K}<T<10^{6.3} \mathrm{~K}$, and the second is valid for $10^{6.3} \mathrm{~K}<T<10^{7} \mathrm{~K}$. We thus construct the grid of numerical models using the radiative-loss function $E_{\mathrm{R} 1}$ for the apex temperature $T_{1}=1 \mathrm{MK}$ and the second radiative-loss function $E_{\mathrm{R} 2}$ for the apex temperatures $T_{1}=3$ and $5 \mathrm{MK}$. Considering $P=1$, the estimated values of $p_{0}$ with respect to the values of $p_{0}^{\left(\sigma_{0}=-1 / 2\right)}$ are

$p_{0}^{\left(\sigma_{1}\right)}=p_{0}^{\left(\sigma_{0}\right)} \cdot 10^{-\frac{6}{4}} \cdot \frac{0.826}{0.716} \sqrt{\frac{3 \times 10^{-31.81}}{2 \times 10^{-34.94}}} \doteq 1.641 p_{0}^{\left(\sigma_{0}\right)}$,

$p_{0}^{\left(\sigma_{2}\right)}=p_{0}^{\left(\sigma_{0}\right)}\left(3 \times 10^{6}\right)^{\frac{1}{12}} \cdot \frac{0.687}{0.716} \sqrt{\frac{5 \times 10^{-31.81}}{9 \times 10^{-30.73}}} \doteq 0.874 p_{0}^{\left(\sigma_{0}\right)}$,

$p_{0}^{\left(\sigma_{2}\right)}=p_{0}^{\left(\sigma_{0}\right)}\left(5 \times 10^{6}\right)^{\frac{1}{12}} \cdot \frac{0.687}{0.716} \sqrt{\frac{5 \times 10^{-31.81}}{9 \times 10^{-30.73}}} \doteq 0.913 p_{0}^{\left(\sigma_{0}\right)}$,

for the cases of $T_{1}=1,3$, and $5 \mathrm{MK}$, respectively. The estimated ratios of base heating rates for these three temperatures are $1.330,0.919$, and 0.919 .

The results of numerical simulations using the two radiativeloss functions $E_{\mathrm{R} 1}$ and $E_{\mathrm{R} 2}$ are depicted in Figs. 5 and 6. It can be seen that for the case of $E_{\mathrm{R} 2}$, the pressures $p_{0}^{\left(\sigma_{2}\right)}$ are close to their estimated values given by Eq. (39), while for $E_{\mathrm{R} 1}$, which deviates more significantly from the case $\sigma_{0}=-1 / 2$, the results for $p_{0}^{\left(\sigma_{1}\right)}$ are slightly further from the expected value. In all
Table 2. Coefficients in scaling laws (24) and (25) reconstructed from values in Table 1.

\begin{tabular}{lllllll}
\hline \hline$T_{1}[\mathrm{MK}]$ & $\beta$ & $\beta^{\prime \prime}$ & $\beta^{\prime \prime \prime}$ & $\gamma$ & $\gamma^{\prime \prime \prime}$ & $\frac{\gamma-\gamma^{\prime \prime \prime}}{\beta-\beta^{\prime \prime \prime}}$ \\
\hline 1 & -0.088 & -0.224 & 0.329 & 0.709 & 0.163 & -1.310 \\
3 & +0.005 & -0.491 & 0.080 & 0.829 & 0.738 & -1.210 \\
5 & +0.013 & -0.708 & 0.067 & 0.861 & 0.807 & -0.989 \\
10 & +0.037 & -0.771 & 0.189 & 0.778 & 0.678 & -0.657 \\
\hline
\end{tabular}

cases, the differences from the expected values given by Eq. (39) increases with increasing $L / s_{\mathrm{H}}$ ratio.

The values of the ratios $E_{\mathrm{H} 0}^{\left(\sigma_{1}\right)} / E_{\mathrm{H} 0}^{\left(\sigma_{0}\right)}$ and $E_{\mathrm{H} 0}^{\left(\sigma_{2}\right)} / E_{\mathrm{H} 0}^{\left(\sigma_{0}\right)}$ are wideranging, making the expected ratio values only a rough estimate. There are large deviations from the expected value for both strongly non-uniform heated loops $\left(L / s_{\mathrm{H}} \gtrsim 1\right)$ and large loops $(L \gtrsim 200 \mathrm{Mm})$. The ratio of base heating rates for the large loops approaches unity for all three studied apex temperatures.

The discrepancies between results of numerical models and the expected values can be explained only in terms of the dependence of the $T(s)$ function on the assumed radiative-loss function. For the fixed values of $T_{1}, L$ and $s_{\mathrm{H}}$, any change in the $T(s)$ function will result in adjustments to the parameters $P, P^{\prime}$, $\beta_{1}, \beta_{2}, \gamma_{1}, \gamma_{2}$ and then implicitly in $p_{0}$ and $E_{\mathrm{H} 0}$. It can then be expected that the discrepancies will be in general larger for the heating rate ratios than for the base pressure ratios, because $\left|\gamma_{2}\right| \gg\left|\gamma_{1}\right|,\left|\beta_{2}\right| \gg\left|\beta_{1}\right|$ (Table 1). However, the exact dependence of the $T(s)$ function on assumed parameters of the radiative-loss function is not known except for the cases of uniform pressure and heating rate (Kuin \& Martens 1982) and uniform pressure and heating explicitly dependent on temperature (Martens 2009). The reconstruction of the temperature profile from Eq. (18) is difficult because of the unknown dependence of $\beta^{\prime}$ and $\gamma^{\prime}$ on $T^{\prime}$. We are thus left with direct construction of grids of numerical models in evaluating the effect of the radiative-loss function on the resulting distributions of $p_{0}$ and $E_{\mathrm{HO}}$.

\subsection{Scaling law for apex temperature}

In this section we return to Eq. (24), which gives the apex temperature as a function of $L, s_{\mathrm{H}}$, and $E_{\mathrm{H} 0}$. The choice of $L, s_{\mathrm{H}}$, and $E_{\mathrm{H} 0}$ as independent parameters is more natural from the perspective of modeling coronal emission in EUV and soft X-ray (e.g., Schrijver et al. 2004), since the coronal emission in the EUV or soft X-ray filter is given by the product of $n_{\mathrm{e}}^{2}$ and the filter response (Mok et al. 2005, and references therein), the latter being a function of both the temperature and electron density.

The values of $\beta, \beta^{\prime \prime}$, and $\gamma$ appearing in Eqs. (24) and (25) together with the values of $\beta^{\prime \prime \prime}$ and $\gamma^{\prime \prime \prime}$ were reconstructed from the values of $P^{\prime}, \beta_{1}, \beta_{2}, \gamma_{1}$, and $\gamma_{2}$ and are listed in Table 2 .

Using the numerically obtained values of $E_{\mathrm{H} 0}$, it is possible to reconstruct the apex temperature $T_{1}$ as function of $L, s_{\mathrm{H}}$, and $E_{\mathrm{H} 0}$. The results are shown in Fig. 7 , which suggests that the resulting apex temperatures $T_{1}$ are within $20 \%$ of their expected values.

We note that the expression $\left(\gamma-\gamma^{\prime \prime \prime}\right) /\left(\beta-\beta^{\prime \prime \prime}\right)$ is negative for all studied apex temperatures. The inequality sign in the condition (23) must then be reversed, so that it is always satisfied.

\subsection{Loops with expanding cross-section}

We now focus our attention on the effect of the expanding crosssection of the loop. We examine in particular the concept of a 


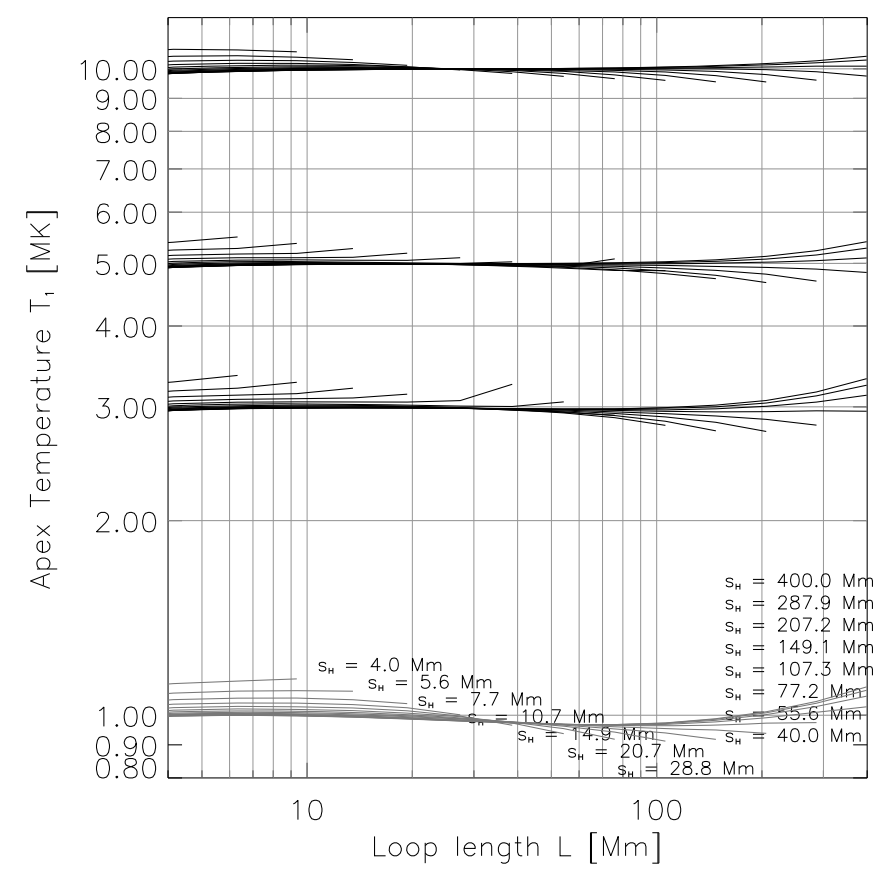

Fig. 7. Scaling law for the apex temperature $T_{1}$ as function of $L, s_{\mathrm{H}}$ and $E_{\mathrm{H} 0}$ (Eq. (24)) reconstructed from the values of Table 2. Points for which the numerical simulations did not converge have been removed form the plot.

loop as an expanding magnetic fluxtube. We utilized the geometry of expanding loops with a buried dipole of Aschwanden \& Schrijver (2002), which generalizes the expanding loop model of Vesecky et al. (1979) for an arbitrary large range of loop expansion factors $\Gamma$. The loop expansion factor is defined to be $\Gamma=A_{1} / A_{0}$, where $A_{1}$ and $A_{0}$ are the apex and photospherical cross-sections of the loop, respectively. The geometrical model of the expanding loop of Aschwanden \& Schrijver (2002) employs the semi-circular geometry in an expanding toroidal shell around the central, semi-circular magnetic field line or loop strand. The central vertical cut through the loop containing the central magnetic field line is depicted in Fig. 3.14 right panel of Aschwanden (2004).

Previous authors (Vesecky et al. 1979; Aschwanden \& Schrijver 2002; Martens 2009) claimed that the effect of expanding loop cross-section on the temperature profile $T(s)$ is small. However, after considering the expanding geometry of Aschwanden \& Schrijver (2002), it is clear that the inward (lowermost) and outward (topmost) field lines or loop strands of the expanding fluxtube have different lengths, and because of Eqs. (24) and (25) they must have different apex temperatures and base pressures; i.e., $T_{1, \mathrm{i}} \neq T_{1, \mathrm{o}}, p_{0, \mathrm{i}} \neq p_{0, \mathrm{o}}$. This means that the emission from these two field lines observed in EUV or X-ray filters must differ. To study this, we computed the ratio of the inward loop strand to the outward loop strand apex emissions in two TRACE filters, 171 and $195 \AA$.

The length $L_{0, \mathrm{i}}$ and $L_{0, \mathrm{o}}$ of the coronal portion of the inward and outward loop strands are given by

$$
\begin{aligned}
& L_{0, \mathrm{i}}\left(\Gamma, A_{0}\right)=\frac{\pi}{2} r_{\mathrm{i}}-2 \arcsin \left(\frac{h_{0}-h_{\mathrm{i}}}{r_{\mathrm{i}}}\right), \\
& L_{0, \mathrm{o}}\left(\Gamma, A_{0}\right)=\frac{\pi}{2} r_{\mathrm{o}}+2 \arcsin \left(\frac{h_{\mathrm{o}}-h_{0}}{r_{\mathrm{o}}}\right),
\end{aligned}
$$

where $r_{\mathrm{i}}=r-\rho-h_{\mathrm{i}}, r_{\mathrm{o}}=r+\rho-h_{\mathrm{o}}$ are the radii of the circles corresponding to these loop strands, $r=2 L / \pi, \rho=\sqrt{\Gamma A_{0} / \pi}$, and $h_{\mathrm{i}}$ and $h_{\mathrm{o}}$ are the heights of the centers of these circles above the photosphere. They are given by

$$
\begin{aligned}
& h_{\mathrm{i}}=\frac{\rho^{2}-2 r \rho}{2(r-\rho)-2 r \sin \left(\phi_{\mathrm{s}}\right)}, \\
& h_{\mathrm{o}}=\frac{\rho^{2}+2 r \rho}{2(r+\rho)-2 r \sin \left(\phi_{\mathrm{s}}\right)},
\end{aligned}
$$

where $\phi_{\mathrm{s}}=-\pi s_{\mathrm{sub}} / 2 L$, and the value of $s_{\mathrm{sub}}(\Gamma)$ is given by Eq. (16) of Aschwanden \& Schrijver (2002). We computed the emission ratios for the values of $\Gamma \in\langle 2,50\rangle, A_{0} \approx 0.53 \mathrm{Mm}^{2}$ and $2.07 \mathrm{Mm}^{2}$ corresponding to $1^{\prime \prime} \times 1^{\prime \prime}$ (TRACE resolution, Handy et al. 1999) and $1.98^{\prime \prime} \times 1.98^{\prime \prime}(\mathrm{SOHO} / \mathrm{MDI}$ resolution, Scherrer et al. 1995), respectively.

We assume that $L_{0}$ is the length of the coronal portion of the central strand and the heating parameters $E_{\mathrm{H} 0}$ and $s_{\mathrm{H}}$ do not change for the entire loop. The computation of the emission ratio proceeds as follows. First, we choose the apex temperature of the central loop strand $T_{1, \mathrm{c}}$ from the interval $\langle 1 \mathrm{MK}, 1.5 \mathrm{MK}\rangle$. Equation (27) then gives the base heating rate $E_{\mathrm{H} 0}$ for the chosen central strand apex temperature. The lengths $L_{0, \mathrm{i}}$ and $L_{0, \mathrm{o}}$ are then computed using Eqs. (40). These values were inserted into Eqs. (24) and (25), which provide the apex temperatures $T_{1, \mathrm{i}}, T_{1, \mathrm{o}}$ and the base pressures $p_{0, \mathrm{i}}$ and $p_{0, \mathrm{o}}$ as functions of $\Gamma, A_{0}, T_{1, \mathrm{c}}, L$, and $s_{\mathrm{H}}$. The apex densities $n_{1, \mathrm{i}}$ and $n_{1, \mathrm{o}}$ were computed using Eqs. (14), (36), (4), and (1). The computed values of $T_{1, \mathrm{i}}, T_{1, \mathrm{o}}$, $n_{1, \mathrm{i}}$, and $n_{1, \mathrm{o}}$ were then used together with the TRACE filter response (Mok et al. 2005) for 171 and $195 \AA$ filters to obtain the emissions in the corresponding filter.

The emission ratio is expected to increase with increasing $\Gamma$, $A_{0}$, and decreasing $L_{0}$, because of the increasing difference between $L_{0, \mathrm{i}}$ and $L_{0, \mathrm{o}}$. We also note that if the apex temperature $T_{1, \mathrm{c}}$ of the central strand is close to the temperature for which the filter response reaches its maximum $(\approx 1 \mathrm{MK}$ for the $171 \AA$ filter, and $\approx 1.5 \mathrm{MK}$ for the $195 \AA$ filter $)$, then the computed emission ratio should be close to unity; this is because the apex temperatures of the inward and outward strands $T_{1, \mathrm{i}}$ and $T_{1, \mathrm{o}}$ will correspond to roughly equal values of the filter response. The situation will be different for temperatures where the filter response is decreasing $\left(T_{1, \mathrm{c}}>1 \mathrm{MK}\right.$ for $171 \AA$ filter $)$ or increasing $\left(T_{1, \mathrm{c}}<1.5 \mathrm{MK}\right.$ for $195 \AA$ filter), where the difference between the $T_{1, \mathrm{i}}$ and $T_{1, \mathrm{o}}$ would increase the observed emission ratio.

We present the results for the case of uniform heating ( $L=$ $40 \mathrm{Mm}, s_{\mathrm{H}}=400 \mathrm{Mm}$; Fig. 8 top row) and non-uniform heating ( $L=40 \mathrm{Mm}, s_{\mathrm{H}}=15 \mathrm{Mm}$; Fig. 8 bottom row). For both these cases, the ratio of the computed emission is too low to explain the observed narrowness of coronal loops (Klimchuk 2000; Watko \& Klimchuk 2000; López Fuentes et al. 2006). The only case where the emission ratio could suffice to explain the observed narrowness of the coronal loops is the case of short ( $L \approx 4 \mathrm{Mm}$ ), uniformly heated loops, since the ratio reaches a factor of $\approx 5$ or more for these loops. We thus conclude that in general the difference in lengths of the inward and outward loop strands cannot explain the observed spatial profile of coronal loops. For stationary, near-potential coronal loops obeying the scaling laws, the parameters of the heating function within a magnetic fluxtube with photospheric cross-sections corresponding to the TRACE resolution ( $\left.1^{\prime \prime}\right)$ must vary rapidly to produce a coronal loop with the observed narrowness. 

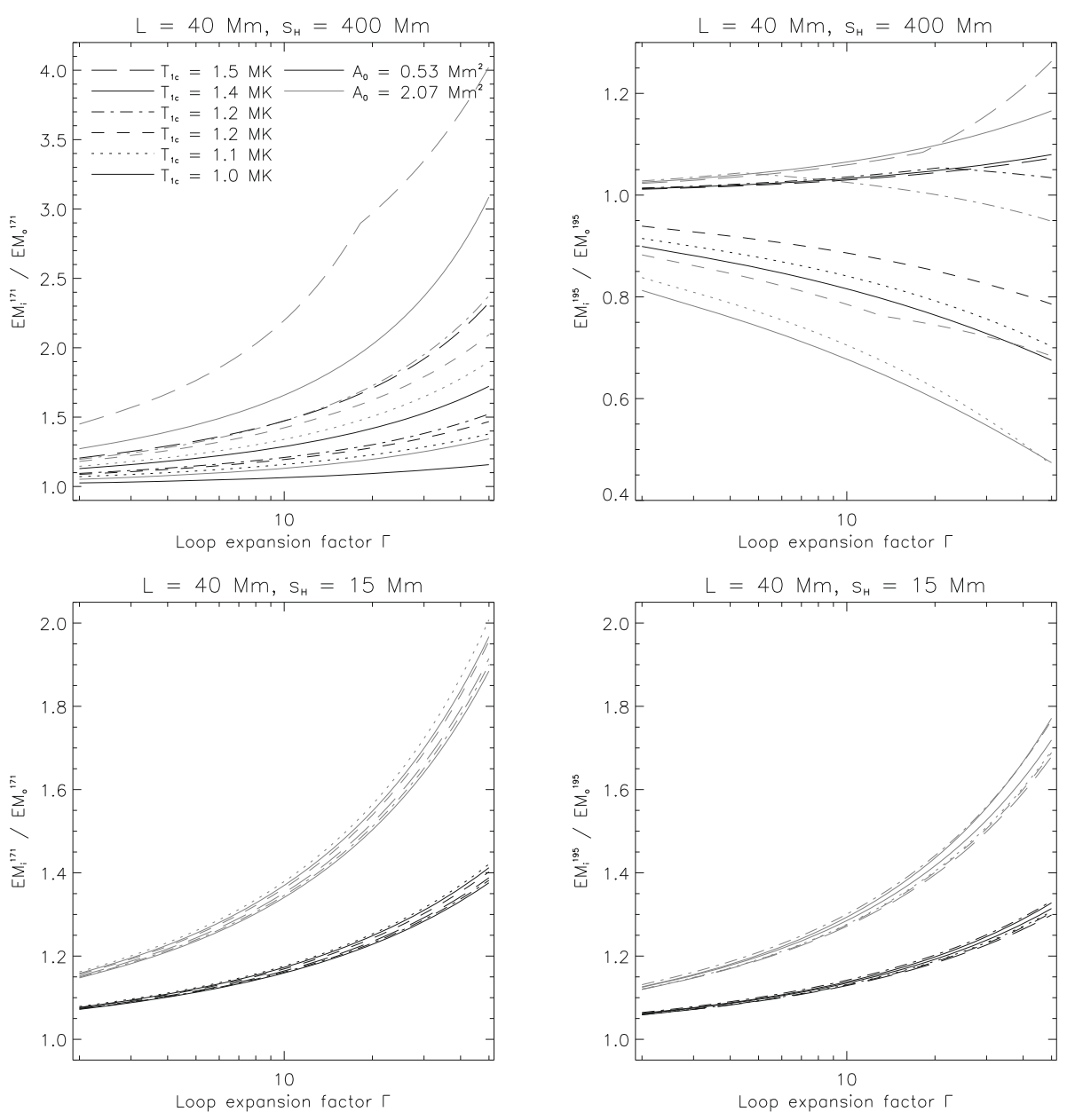

Fig. 8. Computed ratios of emission at apexes of the inward (i) and outward (o) loop strands as function of $\Gamma$ and $A_{0}$. Left column: emission ratios for the $171 \AA$ TRACE filter, Right column: emission ratios for the $195 \AA$ TRACE filter. Top row: Case of uniform heating, Bottom row: case of non-uniform heating.

\section{Conclusions}

We have derived an analytical set of scaling laws between loop apex temperature $T_{1}$, base pressure $p_{0}$, base heating rate $E_{\mathrm{H} 0}$, loop half-length $L$, and heating scale length $s_{\mathrm{H}}$ for geometrically symmetrical, non-uniformly heated, and gravitationally stratified one-dimensional loop strands that are thermally isolated with monotonically increasing temperature profile. For analytical treatability, the derivation of the scaling laws employed the mean-value theorem, which directly implied the need of fitting the analytical results to the numerical simulations under the assumption of constant mean-value parameters. This strict assumption in turn leads to moderate accuracy of the scaling laws with respect to the numerical simulations. Higher accuracy can be achieved only by employing Taylor expansion or additional empirical terms. The latter provides higher precision, and ensures that the scaling laws of Aschwanden \& Schrijver (2002) are the most precise scaling laws existing to date, even though their empirical terms have no analytical background. However, the presence of $d_{0}$ and $e_{0}$ parameters is justified in terms of our parameter $P^{\prime}$ and the $I(\sigma, P)$ function.

We performed a case study of the semi-elliptical loop geometries. The difference in loop geometry results only in relatively small changes in $p_{0}$ and $E_{\mathrm{H} 0}$, of the order of less than approximately $6 \%$ for $p_{0}$ and about twice as much for $E_{\mathrm{H} 0}$. The results are more sensitive to the parameters of the radiativeloss function. The base pressure $p_{0}$ depends explicitly on both $\chi$ and $\sigma$, while the dependence of the base heating rate $E_{\mathrm{H} 0}$ on these parameters is apart from the presence of the $I(\sigma, P)$ function by means of the temperature profile. However, the effect of radiative-loss function is non-negligible and thus a correct power-law approximation to the radiative-loss function must be taken before the scaling laws are applied, e.g., in forward modeling of coronal emission. It is interesting that the base heating rate $E_{\mathrm{H} 0}$ for long, uniformly heated loops is almost independent of the details of the radiative-loss function.

The effect of an expanding loop cross-section on the resulting EUV emission in TRACE $171 \AA$ and $195 \AA$ filters was studied. We conclude that the different lengths of the inward and outward loop strand are insufficient to explain the spatial narrowness of coronal loops except for the case of small, uniformly heated loops, i.e., if the parameters of the heating function did not vary in space, both inward and outward loop strands would be visible in a given TRACE EUV filter.

\section{Appendix A: Mean-value theorem}

The mean-value theorem for integration (e.g., Bartsch 1977) states that for the integrable function $f(x)$ and continuous function $g(x), x \in\langle a, b\rangle$, there exists a number $\vartheta \in(a, b)$, for which the following relation holds:

$$
\int_{a}^{b} f(x) g(x) \mathrm{d} x=g(\vartheta) \int_{a}^{b} f(x) \mathrm{d} x .
$$


We demonstrate the use of this theorem in evaluating the integral $J$ defined by (cf. Eq. (15))

$J=\int_{0}^{T_{1}} C T^{\sigma+1 / 2} \exp \left(-\frac{2\left(h(s)-h_{0}\right)}{\lambda_{0} T(s)\left(1+h(s) / R_{\odot}\right) q_{\lambda}}\right) \mathrm{d} T$.

Here we must take into account that because of the assumptions presented in Eqs. (11), (12), and (13), the temperature profile $T(s)$ monotonically increases, which means that there exists a unique and monotonic inverse function $s=s(T)$. Similarly, due to the $h=h(s)$ dependence, there exists a unique $h=h(T)$ function.

Using the mean-value theorem it is clear that there exists some $\xi, 0<\xi<1$ for which the integral (A.2) equals to

$J=\exp \left(-\frac{2\left(h\left(\xi T_{1}\right)-h_{0}\right)}{\lambda_{0} \xi T_{1}\left(1+h\left(\xi T_{1}\right) / R_{\odot}\right) q_{\lambda}}\right) \int_{0}^{T_{1}} C T^{\sigma+1 / 2} \mathrm{~d} T$.

Since the function $h(T)$ is monotonic, there must exist some $\zeta$ and $\eta$, such that $0<\zeta, \eta<1$, and defined by

$$
\begin{aligned}
h\left(\xi T_{1}\right) & =\zeta h_{1}, \\
1+h\left(\xi T_{1}\right) / R_{\odot} & =\eta\left(1+h_{1} / R_{\odot}\right) .
\end{aligned}
$$

Thus the integral $J$ equals to

$$
J=\frac{C T_{1}^{\sigma+3 / 2}}{\sigma+3 / 2} \exp \left(-\beta \frac{h_{1}-h_{0}}{\lambda_{\mathrm{p}}}\right),
$$

where $\lambda_{\mathrm{p}}=\lambda_{0} T_{1}$ and the parameter $\beta$ is defined as

$\beta=\frac{2}{\xi \eta q_{\lambda}} \frac{\zeta h_{1}-h_{0}}{h_{1}-h_{0}} \frac{1}{1+h_{1} / R_{\odot}}$.

Equation (36) can be used to convert Eq. (A.6) into the final result

$J=\frac{C T_{1}^{\sigma+3 / 2}}{\sigma+3 / 2} \exp \left(-\beta \frac{L-s_{0}}{s_{\mathrm{p}}}\right)$.

We note that the $q_{\lambda}$ correction factor depends on apex temperature $T_{1}$, loop half-length $L$, and also on heating scale length $s_{\mathrm{H}}$ (Eq. (27) and Table 1 of Aschwanden \& Schrijver 2002). The temperature profile $T(s)$ can also be dependent on $L$ and $s_{\mathrm{H}}$, which means that the $\xi, \zeta$, and $\eta$ parameters are functions of $T_{1}, L, s_{\mathrm{H}}$, and thus $\beta=\beta\left(T_{1}, L, s_{\mathrm{H}}\right)$.
Acknowledgements. The authors are thankful to the referee, P. Martens, for his comments that helped to clarify several issues in the manuscript. We are indebted to M. Aschwanden for incorporating the hydro package into the SolarSoft library, thus providing us with a powerful and versatile tool to study the equilibrium solutions for coronal loops. We are also thankful for helpful and lengthy discussions regarding the software. We would also like to extend our thanks to J. Klačka for helpful discussion regarding the $I(\sigma, P)$ function. This work was supported by Scientific Grant Agency, VEGA, Slovakia, Grant No. 1/0069/08, Grant IAA300030701 of the Grant Agency of the Academy of Sciences of the Czech Republic and ESA-PECS project No. 98030. J.D. also acknowledges the financial support of the Comenius University Grant No. 414/2008. The Solar and Heliospheric Observatory ( $\mathrm{SOHO}$ ) is a project of international cooperation between ESA and NASA. The Transition Region and Coronal Explorer (TRACE) is a mission of the Stanford-Lockheed Institute for Space Research, and part of the NASA Small Explorer program.

\section{References}

Antiochos, S. K. 1979, ApJ, 232, L125

Aschwanden, M. J. 2004, Physics of the Solar Corona: An Introduction (United Kingdom: Springer, PRAXIS, Chichester)

Aschwanden, M. J., \& Schrijver, C. J. 2002, ApJ, 142, 269

Aschwanden, M. J., \& Tsiklauri, D. 2009, ApJ, submitted

Bartsch, H.-J. 1977, Mathematische Formeln (Germany: VEB Fachbuchverlag, Leipzig)

Chiuderi, C., Einaudi, G., \& Torricelli-Ciamponi, G. 1981, A\&A, 97, 27

Craig, I. J. D., McClymont, A. N., \& Underwood, J. H. 1978, A\&A, 70, 1

Craig, I. J. D., Robb, T. D. \& Rollo, M. D. 1982, Sol. Phys., 76, 331

Freeland, S. L., \& Handy, B. N. 1998, Sol. Phys., 182, 497

Handy, B. N., Acton, L. W., Kankelborg, C. C., et al. 1999, Sol. Phys., 187, 229

Hood, A. W., \& Priest, E. R. 1979, A\&A, 77, 233

Hood, A. W., \& Priest, E. R. 1980, A\&A, 87, 126

Klimchuk, J. A. 2000, Sol. Phys., 193, 53

Klimchuk, J. A. 2006, Sol. Phys., 234, 41

Kuin, N. P. M., \& Martens, P. C. H. 1982, A\&A, 108, L1

López Fuentes, M. C., Klimchuk, J. A., \& Démoulin, P. 2006, ApJ, 639, 459

Marquardt, D. W. 1963, J. Soc. Indust. Appl. Math., 11, 431

Martens, P. C. H. 2009, ApJ, submitted

Martens, P. C. H., Kankelborg, C. C., \& Berger, T. E. 2000, ApJ, 537, 471

Mok, Y., Mikić, Z., Lionello, R., \& Linker, J. A. 2005, ApJ, 621, 1098

Priest, E. R. 1982, Solar Magnetohydrodynamics (Holland: D. Reidel Publishing Company, Dordrecht)

Raymond, J. C., \& Smith, B. W. 1977, ApJS, 35, 419

Rosner, R., Tucker, W. H., \& Vaiana, G. S. 1978, ApJ, 220, 643

Scherrer, P. H., Bogart, R. S., Bush, R. I., et al. 1995, Sol. Phys., 162, 129

Schrijver, C. J., Sandman, A. W., Aschwanden, M. J., \& DeRosa, M. L. 2004, ApJ, 615, 512

Serio, S., Peres, G., Vaiana, G. S., et al. 1981, ApJ, 243, 288

Spitzer, L. 1962, Physics of Fully Ionized Gases (New York: Interscience)

Tucker, W. H., \& Koren, M. 1971, ApJ, 168, 283

Vesecky, J. F., Antiochos, S. K., \& Underwood, J. H. 1979, ApJ, 233, 987

Watko, J. A., \& Klimchuk, J. A. 2000, Sol. Phys., 193, 77

Wragg, M. A., \& Priest, E. R. 1981, Sol. Phys., 70, 293 